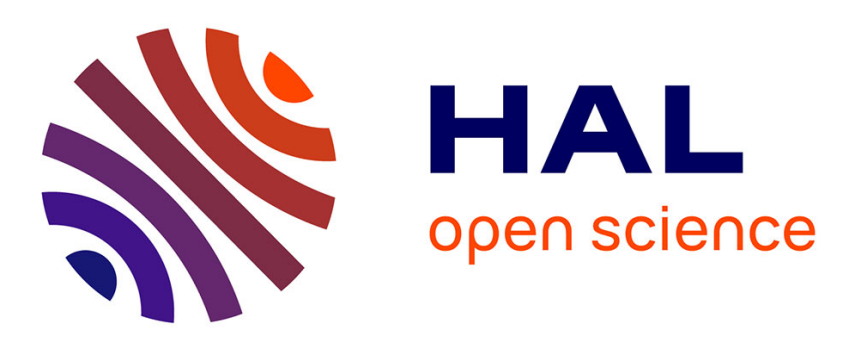

\title{
Path protection in optical flexible networks with distance-adaptive modulation formats
}

Andrea Tomassilli, Brigitte Jaumard, Frédéric Giroire

\section{To cite this version:}

Andrea Tomassilli, Brigitte Jaumard, Frédéric Giroire. Path protection in optical flexible networks with distance-adaptive modulation formats. 2018 International Conference on Optical Network Design and Modeling (ONDM), May 2018, Dublin, France. 10.23919/ONDM.2018.8396102 . hal-01921082

\section{HAL Id: hal-01921082 \\ https://hal.inria.fr/hal-01921082}

Submitted on 14 Nov 2018

HAL is a multi-disciplinary open access archive for the deposit and dissemination of scientific research documents, whether they are published or not. The documents may come from teaching and research institutions in France or abroad, or from public or private research centers.
L'archive ouverte pluridisciplinaire HAL, est destinée au dépôt et à la diffusion de documents scientifiques de niveau recherche, publiés ou non, émanant des établissements d'enseignement et de recherche français ou étrangers, des laboratoires publics ou privés. 


\title{
Path Protection in Optical Flexible Networks with Distance-adaptive Modulation Formats
}

\author{
A. Tomassilli*, B. Jaumard ${ }^{\dagger}$, and F. Giroire* \\ *Université Côte d'Azur, CNRS, Inria Sophia Antipolis, France \\ †Concordia University, Montreal (Qc) Canada
}

\begin{abstract}
Thanks to a flexible frequency grid, Elastic Optical Networks (EONs) will support a more efficient usage of the spectrum resources. On the other hand, this efficiency may lead to even more disruptive effects of a failure on the number of involved connections with respect to traditional networks. In this paper, we study the problem of providing path protection to the lightpaths against a single fiber failure event in the optical layer. Our optimization task is to minimize the spectrum requirements for the protection in the network. We develop a scalable exact mathematical model using column generation for both shared and dedicated path protection schemes. The model takes into account practical constraints such as the modulation format, regenerators, and shared risk link groups. We demonstrate the effectiveness of our model through extensive simulation on two real-world topologies of different sizes. Finally, we compare the two protection schemes under different scenario assumptions, studying the impact of factors such as number of regenerators and demands on their performances.
\end{abstract}

\section{INTRODUCTION}

In an Elastic Optical Network (EON), data is distributed over a number of low data rate subcarriers without having to strictly follow the ITU-T fixed wavelength grid. In this way, with a data traffic more and more uncertain and heterogeneous, the spectrum resources can be used more efficiently and with a higher degree of flexibility [1].

With respect to a classical WDM network, EONs impose additional constraints on the structure of the optical path. Indeed, EONs require that contiguous frequency slots are allocated to each connection, which is also the main difference between the Routing and Spectrum Assignment (RSA) and Routing and Wavelength Assignment (RWA) problems. Thus, the already proposed RWA methods are not suitable for EONs. The RSA problem requires to find both an end-to-end optical path and a contiguous subset of frequency slots for each connection request.

Furthermore, EONs open up the possibility of exploiting multiple modulation formats for the different subcarriers. In such a way, the utilization efficiency could be further enhanced [2]. The problem of also determining a modulation format in addition to a routing path and a contiguous segment of spectrum is often referred to as the Routing, Modulation, and Spectrum Allocation (RMSA) problem. The problem is known to be NP-Hard even in the absence of modulation formats [3] and is challenging, even on small instances.

With the increasing efficiency in terms of resource usage, a link may accommodate a larger number of connections in EONs. Hence, the effects of a failure, such as a fiber cut, could be even more disruptive than in traditional networks. Network failures have been widely investigated (see e.g., [4], [5]). In the results of [4], each link experienced, on average, 16 failures per year. If not well managed, a failure may correspond to loss of service to users and loss of revenue. It is thus necessary to provide protection against failures in order to guarantee continuity of service and no violation of SLA requirements. We focus our attention on the single link failure scenario, since they are the predominant form of failures in optical networks [6].

Fault management techniques can be grouped into two categories: restoration and protection. In restoration, the network spare resources are used to reroute the connections affected by the failure. In protection, spare capacity is reserved in advance during connection setup. Restoration schemes use network spare resources more efficiently, but on the other hand, protection schemes have a faster restoration time and guarantee the recovery [7]. We thus study the latter schema.

In dedicated protection, there is no spectrum resources sharing between backup lightpaths. Each frequency slot is used for at most one lightpath. In shared protection, backup spectrum resources can be shared among different lightpaths if they fail independently. If, on one hand, in shared protection, spectrum resources are used more efficiently [8], on the other hand, in dedicated protection the recovery time is smaller. We thus study both protection schemes in this paper.

Another classification of the protection techniques can be made according to the recovery mechanisms. It could consist in a local repair (i.e., link protection) or in an end-to-end repair (i.e., path protection). Link protection schemes reroute the traffic only around the failed link. Path protection schemes reroute the traffic through a backup path if a failure occurs on its working path. With path protection, network resources are used more efficiently [6].

We consider the problem of providing for each connection, a link-disjoint backup lightpath, under both dedicated and shared path protection schemes. Our model also includes practical parameters such as the modulation format selection and the positions of regenerators. The modulation format of a lightpath adds a constraint on the maximum transmission distance, which may be extended by one or more regenerators if present in the route. One of the key concerns of the network operators is the efficient utilization of the deployed network capacity [1]. Our optimization goal is thus the minimization of the spectrum requirements for the protection.

In this paper, we propose two models for both dedicated and shared path protection against a single link failure. Our resolution strategy is based on a decomposition model using the column generation technique. We show that this technique is effective in dealing with the RMSA problem.

Our contributions can be summarized as follows:

- To the best of our knowledge, we are the first to propose 
a scalable exact method to solve the problem of providing path protection against a single link failure in elastic optical networks. The method is based on a decomposition model using column generation.

- The model takes into account practical constraints, such as multiple modulation formats, regenerators, and shared risk link groups.

- We compare the shared and dedicated path protection models and evaluate the tradeoff between the resolution time and the effectiveness, in terms of bandwidth utilization.

- We additionally study the impact of the number of regenerators in the network on the bandwidth requirements and on the latencies of both primary and backup lightpaths.

The rest of this paper is organized as follows. In Section II, we review related works in more detail. In Section III, we formally state the problem addressed in this paper. In section IV, we describe our column-generation-based model and show the subproblem to be solved in Section V. In Section VI, we validate our model by various numerical results on two real world topologies of different sizes. Finally, we draw our conclusions in Section VII.

\section{RELATED WORK}

The problem of providing protection against failures in WDM networks has been widely investigated in the literature, see e.g., [6], [7], [8]. Nevertheless, not enough effort has been made in the context of EONs with multiple modulation formats and flexible spectrum allocation.

Dedicated path protection. The problem of off-line routing and spectrum allocation in flexible grid optical networks with dedicated path protection was studied in [9] and [10]. The optimization goal considered is to minimize the width of spectrum required in the network. In [9], the authors provide both an ILP formulation and a heuristic algorithm to solve the problem. In [10], an evolutionary algorithm metaheuristic is proposed with the aim to support the search for optimal solutions.

Shared path protection. Shared protection for EONs was considered in [11], [12], and [13]. A genetic algorithm metaheuristic with the goal to provide near optimal solutions to the problem of finding a primary and a backup path for each demand is proposed in [11]. The closest works to ours are [12] and [13]. The authors consider exact methods and propose ILP formulations for both dedicated and shared path protection, but with different optimization objectives. In [12], the authors minimize both the required spare capacity and the maximum number of frequency slots used in the network. In [13], the objective is to minimize the width of spectrum required in the network. They propose an ILP formulation in which each demand has a set of candidate pairs of link disjoint routing paths. The ILP model is able to deal with small networks (up to 9 nodes and 26 links). For larger networks, they propose heuristic algorithms based on both jointly and separated assignment of lightpaths to the demands.

Model Scalability. Previous works highlight the fact that finding an optimal or a near-optimal solution to the problem of jointly computing both a primary and a backup path for each demand is a challenging task, even for networks of small sizes and for a small number of demands. For instance, in [13] the authors show the benefits in terms of computing time and accuracy of computing the set of backup paths after the

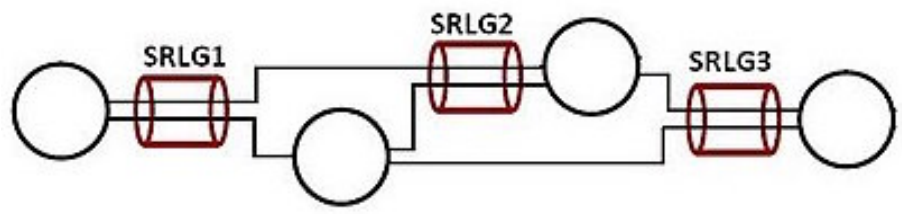

Fig. 1: An example of SRLG Constraints

primary path allocation. In order to be able to deal with larger datasets, we adopt a two phase approach. First, we find a working path for each demand, then a backup path under both dedicated and shared protection schemes. We use the column generation technique as a solution approach, as results from [14] evidence the effectiveness of the column generation techniques in obtaining solutions for large instances of the RSA problem (but they do not consider protection against failures). Exact models proposed in the literature are only able to deal with small networks. On the contrary, our model is more scalable and we are able to solve instances with 24 nodes, 43 links, and 120 traffic requests. Moreover, we take into account regenerators and choices of modulation formats, which are not considered in the exact models of the literature.

\section{Statement of the RMSA Protection Problem}

The RMSA problem assumes an undirected graph $G=$ $(V, L)$ with optical node set $V$ and link set $L$. We denote by $\omega(v)$ the set of links adjacent to $v$, for $v \in V$. The bandwidth is slotted into a set $S$ of frequency slots. The traffic is defined by a set $K$ of requests where each request $k \in K$ has a source $\left(s_{k}\right)$, a destination $\left(d_{k}\right)$, and a spectrum demand $D_{k}$, expressed in terms of a number of frequency slots. The traffic is assumed to be symmetrical.

The provisioning of the primary lightpaths is given, and we are interested in finding both a dedicated and a shared path protection with minimum spectrum requirements, satisfying the spectrum contiguity and continuity constraints, as well as the following constraints:

- Shared Risk Link Group (SRLG) constraints, see Figure 1. Each SRLG constraint is defined by a set of links sharing a common resource, which affects all links in the set if the common resource fails. In the context of optical networks, it refers to a bundle of fiber links going through the same duct and that cannot be used simultaneously for primary and backup provisioning of the same demand. Let $\mathcal{F}$ be the set of all SRLG sets: $\mathcal{F}=\left\{F\right.$ : if $\ell$ and $\ell^{\prime}$ both belong to $F$, then $\ell$ cannot be used in a path protecting $\ell^{\prime}$ and vice versa $\}$.

- Modulation constraints The modulation format can be selected according to the traffic demand and the distance. We consider four modulation formats: BPSK (1 bit per symbol), QPSK (2 bits per symbol), 8QAM (3 bits per symbol), and 16QAM (4 bits per symbol) [15]. For instance, if, for a demand $k$, we have a request of $250 \mathrm{~Gb} / \mathrm{s}$ (i.e., $D_{k}=20$ assuming the bandwidth of a subcarrier slot as $12.5 \mathrm{GHz}$ ), then with BPSK $D_{k}^{B S P K}=20$ and with 16QAM, $D_{k}^{16 Q A M}=5$. We consider the following maximum transmission distances: BPSK $(9,600$ $\mathrm{Km})$, QPSK $(4,800 \mathrm{Km})$, 8QAM $(2,400 \mathrm{Km})$, and 16QAM $(1,200 \mathrm{Km})$. These values are based on the experimental results reported in [16]. Moreover, we assume that a subset of the nodes have regeneration capabilities. Indeed, decisions about 
the required equipment (i.e., transponders, regenerators, and switches) and its deployment are taken during the planning phase [17].

\section{Path Protection Models}

We propose two column generation models relying on lightpath configurations for both dedicated and shared path protection schemes. In the rest of the paper, the two models will be referred to, respectively, as CG_DP and CG_SP.

A lightpath configuration, denoted by $\pi$, refers to a backup lightpath, i.e., a backup path, a spectrum slice with $s$ as a starting frequency slot and a modulation format. Denote by $\Pi$ the set of all possible backup lightpath configurations. $\Pi$ is decomposed as follows:

$$
\Pi=\bigcup_{k \in K} \Pi_{k}=\bigcup_{k \in K} \bigcup_{s \in S} \Pi_{k s},
$$

where $\Pi_{k}$ is the set of potentials lightpaths for provisioning request $k$, and $\Pi_{k s}$ is the set of potential lightpaths for provisioning request $k$ with a slot slice of width $D_{k}^{m}$ according to the selected modulation format $m$ such that $s$ is a starting slot. Note that $\Pi_{k}$ contains only feasible backup lightpaths for a demand $k$. We say that a backup lightpath is feasible for $k$ if it does not contain any link in the same shared risk link group of some link of the primary lightpath for $k$. Each lightpath configuration, or lightpath for short, is denoted by $\pi$ and is characterized by:

$b_{\ell s}^{\pi}$ : indicates if slot $s$ is used on link $\ell$ in the backup lightpath associated with $\pi$.

We assume that working lightpaths are known and described throughput the following parameter:

$a_{\ell}^{k}$ : indicates if the primary lightpath of request $k$ goes through link $\ell$.

The model uses the following decision variables:

$z_{\pi}=1$ if lightpath $\pi \in \Pi$ is selected as a backup path, 0 otherwise.

$x_{\ell s}=1$ if slot $s$ is used on link $\ell$ in a backup path, 0 otherwise. We denote with $\mathcal{L} S^{\mathrm{B}}$ the pairs $(\ell, s) \mid \ell \in L, s \in S$ that can be used for protection, i.e., that are not used by the primary lightpaths.

The objective minimizes the spectrum requirements for the protection, and is written as follows:

$$
\min \sum_{(\ell, s) \in \mathcal{L} S^{\mathrm{B}}} x_{\ell s}
$$

Constraints are as follows:

$$
\begin{array}{ll}
\sum_{\pi \in \Pi_{k}} z_{\pi} \geq 1 & k \in K \\
z_{\pi} \in\{0,1\} & \pi \in \Pi \\
x_{\ell s} \in\{0,1\} & \ell \in L, s \in S
\end{array}
$$

\section{Model CG_DP}

$$
\sum_{k \in K} \sum_{\pi \in \Pi_{k}} b_{\ell s}^{\pi} z_{\pi} \leq x_{\ell s} \quad \ell \in L, s \in S,(\ell, s) \in \mathcal{L} S^{\mathrm{B}}
$$

\section{Model CG_SP}

$$
\begin{aligned}
\sum_{k \in K} a_{\ell^{\prime}}^{k} \sum_{\pi \in \Pi_{k}} b_{\ell s}^{\pi} z_{\pi} \leq x_{\ell s} \quad \ell, \ell^{\prime} \in L, s \in S \\
\left\{\ell, \ell^{\prime}\right\} \nsubseteq F: F \in \mathcal{F}, \ell \neq \ell^{\prime},(\ell, s) \in \mathcal{L} S^{\mathrm{B}}
\end{aligned}
$$

Constraint (2) ensures that each request is protected. Constraints (5) and (6) make sure that each slot is never used more than once on each backup fiber link. The difference between the two models relies on these constraints. In the dedicated protection case, two working paths cannot have backup paths going through the same link $\ell$ and slot $s$. On the other hand, in the shared protection case, two working paths that are not sharing any link $\ell^{\prime}$ can use protection paths going through the same link $\ell$ and slot $s$.

\section{Solution Design}

Given the huge number of variables/columns in the proposed model, we resort to the Column Generation method to solve its Linear Programming (LP) relaxation, see, e.g., Chvatal [18], if not familiar with this technique. This technique consists of decomposing the original problem into a restricted master problem - RMP - (i.e., model (1) - (6) with a very restricted number of variables) and one or several pricing problems - PPs. RMP and PPs are solved alternately. Solving RMP consists in selecting the best lightpaths, while solving one PP allows the generation of an improving potential lightpath, i.e., a lightpath such that, if added to the current RMP, improves the optimal value of its LP relaxation. The process continues until the optimality condition is satisfied, that is, the so-called reduced cost that defines the objective function of the pricing problems is non negative for all of them. An $\varepsilon$-optimal solution for the RSA problem is derived by solving exactly the ILP model associated with the last RMP.

Let $K_{\sigma}$ denote the set of requests that have the potential to be protected by a lightpath starting at slot $\sigma: K_{\sigma}=\{k \in K$ : $\left.\sigma+D_{k}-1 \leq|S|\right\}$. Let $D_{k}^{\sigma}$ be the number of slots needed for request $k$ in $K_{\sigma}: D_{k}^{\sigma}=D_{k}$ for $k \in K_{\sigma}: \sigma+D_{k}-1=|S|$ and $D_{k}^{\sigma}=D_{k}+1$ for $k \in K_{\sigma}: \sigma+D_{k}-1<|S|$.

Each pricing problem is indexed by a demand $k$ and a starting slot $\sigma$, and produces a single potential lightpath for protecting demand $k$, starting at slot $\sigma$.

Definitions of the decision variables are as follows:

$y_{\ell}=1$ if link $\ell$ is used, 0 otherwise

$x_{\ell s}$ indicates if slot $s$ is used on link $\ell$ or not.

We first describe the model for shared protection. Let $u_{k}^{(2)}$ and $u_{\ell \ell^{\prime} s}^{(6)}$ be the values of the dual variables associated with constraints (2) and (6), respectively. The pricing problem can be written as follows:

$$
\min 0-u_{k}^{(2)}-\sum_{(s, \ell) \in S \times L} \sum_{\substack{\ell^{\prime} \in L: \\ \ell \neq \ell^{\prime}}} u_{\ell \ell^{\prime} s}^{(6)} a_{\ell^{\prime}}^{k} x_{\ell s}
$$

subject to:

$$
\begin{array}{ll}
\sum_{\ell \in \omega\left(s_{k}\right)} y_{\ell}=\sum_{\ell \in \omega\left(d_{k}\right)} y_{\ell}=1 & v \in V \backslash\left\{s_{k}, d_{k}\right\} \\
\sum_{\ell \in \omega(v)} y_{\ell} \leq 2 & v \in V \backslash\left\{s_{k}, d_{k}\right\}, \ell \in \omega(v) \\
\sum_{\ell^{\prime} \in \omega(v) \backslash\{\ell\}} y_{\ell^{\prime}} \geq y_{\ell} & \ell \in L \\
\sum_{s=\sigma}^{\sigma+D_{k}^{\sigma}-1} x_{\ell s}=D_{k}^{\sigma} y_{\ell} & \ell \in L, s \in S
\end{array}
$$


Constraints (8), (9) and (10) define the routing of the current request. Constraint (11) reserves a contiguous spectrum channel for the current request.

We observe that for each link $\ell$ :

$x_{\ell s}=y_{\ell}$ for $s \in\left\{\sigma, \ldots, \sigma+D_{k}^{\sigma}-1\right\}$

$x_{\ell s}=0$ for $s \notin\left\{\sigma, \ldots, \sigma+D_{k}^{\sigma}-1\right\}$.

Therefore, the reduced cost can be rewritten:

$$
\min \quad 0-u_{k}^{(2)}-\sum_{\ell \in L}\left(\sum_{\substack{\ell^{\prime} \in L: \\ \ell \neq \ell^{\prime}}} \sum_{s=\sigma}^{\sigma+D_{k}^{\sigma}-1} u_{\ell \ell^{\prime} s}^{(6)}\right) y_{\ell} .
$$

The first term is a constant for each request, and the second term corresponds to a summation over the links of the network. Therefore, we can solve the pricing problem using the following objective function:

$$
\min -\sum_{\ell \in L}\left(\sum_{\substack{\ell^{\prime} \in L: \\ \ell \neq \ell^{\prime}}} \sum_{s=\sigma}^{\sigma+D_{k}^{\sigma}-1} u_{\ell \ell^{\prime} s}^{(6)}\right) y_{\ell}
$$

where $u_{\ell \ell^{\prime} s}^{(6)}$ are non-positive dual values. We conclude that, for each request $k$, the lightpath generator corresponds to a weighted shortest-path problem with link weight: $-\sum_{\ell^{\prime} \in L: \ell \neq \ell^{\prime}} \sum_{s=\sigma}^{\sigma+D_{k}^{\sigma}-1} u_{\ell \ell^{\prime} s}^{(6)}$. As a result, the pricing problem when modulation and regenerators are not taken into account can be solved with a polynomial time algorithm, e.g., Dijkstra's algorithm.

In the dedicated protection case, the only difference lies in the objective function of the pricing problem, defined as:

$$
\min \quad 0-u_{k}^{(2)}-\sum_{(s, \ell) \in S \times L} u_{\ell s}^{(5)} x_{\ell s}
$$

where $u_{k}^{(2)}$ and $u_{\ell s}^{(5)}$ are the values of the dual variables associated with constraints (2) and (5), respectively. As with the shared protection case, the problem can be reduced to finding a shortest path in a weighted graph.

Additional Modulation and Regenerators Constraints. However, if modulation is taken into account, we need to consider the maximum transmission distance constraint according to the considered modulation format. Also, a regenerator may extend the maximum reachable distance with respect to the chosen modulation format.

Each pricing problem is now indexed by a demand $k$, a starting slot $\sigma$, and a modulation format $m$, and produces a single potential lightpath for protecting demand $k$, starting at slot $\sigma$, if such a lightpath exists. In fact, some demands may not be satisfied, since the reachable distance is not long enough to reach the destination from the source, even in the presence of regenerators.

Regenerators add an additional layer of complexity to the problem. Indeed, without regenerators, for a demand $(s, t)$, we could only consider to solve the subproblem for the modulation formats whose transmission reach is greater or equal to the length of the shortest path between $s$ and $t$. With the presence of regenerators, this consideration does not apply, since the transmission reach may be increased.
When considering modulation constraints and nodes with regenerator capabilities, the pricing problem becomes a Minimum-Weight Path Problem with a constraint on the path length. The Minimum-Weight Constrained Path Problem is proven to be NP-Hard [19]. The problem has been widely studied and efficient algorithms have been proposed (see [20] for a survey on the subject).

Our solving strategy is described as follows. Pricing problems are solved using a modified version of the Label-setting algorithm for the Shortest Path Problem with Resource Constraints [20] based on the dynamic programming approach. Given a weighted graph $G=(V, E)$, a demand $(s, t)$, the maximum transmission distance according to the selected modulation format, and a set of nodes with regenerator capabilities $V_{r} \in V$, the algorithm starts from the trivial path $P=(s)$. It is then extended in all the feasible directions considering both the length of the links and the remaining transmission distance from the source $s$, which may have been increased because of the presence of one or more nodes in the set $V_{r}$ in the considered path. For each path extension $P^{\prime} \supset P$, a dominance algorithm is used in order to maintain only a Pareto-optimal set of paths or paths which can be extended to a Pareto-optimal one. When there are no more labels to be processed, the algorithm stops. A solution of minimum cost is selected from the set of all computed paths.

\section{NUMERICAL RESULTS}

In this section, we evaluate the accuracy and performance of the proposed models through simulation on two networks of different sizes and according to different types of metrics. The results indicate that our models perform well, with an accuracy better than $1 \%$ for CG_DP and $20 \%$ for CG_SP in the considered networks. We also compare the performance of the dedicated and shared protection schemes, and show the tradeoff between the time needed to find a solution to the problem in the two cases and the savings in terms of bandwidth overhead.

Data Sets. We conduct experiments on two network topologies: nobel-US (14 nodes, 21 links) from SNDlib [21], and USnet (24 nodes, 43 links) from [22]. For nobel-US, the length of each link is calculated using the GPS coordinates of the nodes, according to the Cosine-Haversine formula. We assume that there is one pair of bidirectional fibers on each link, and the available spectrum width of each fiber is set to be $2000 \mathrm{GHz}$. We set the bandwidth of a subcarrier slot to 12.5 $\mathrm{GHz}$. We considered four modulation formats: BPSK (binary phase-shift keying), QPSK (quadrature phase-shift keying), 8QAM (8-quadrature amplitude modulation), and 16QAM (16quadrature amplitude modulation). Similarly, as in [23], we assume transmission distances of $9,600 \mathrm{~km}$ for BPSK $(\mathrm{M}=$ 1), 4,800 km for QPSK ( $\mathrm{M}=2), 2,400 \mathrm{~km}$ for 8QAM (M = $3)$, and $1,200 \mathrm{~km}$ for $16 \mathrm{QAM}(\mathrm{M}=4)$, where $\mathrm{M}$ denotes the number of bits per symbol. The number of considered nodes with regenerator capabilities is 5 for nobel-US and 10 for USnet. Locations are chosen according to the betweenness centrality, an index of the importance of an element in the network. It measures the extent to which a node lies on paths between other nodes. Primary paths are computed with the objective of minimizing the total number of used frequency slots in the network. All experiments are run on an Intel Xeon E5520 with 24GB of RAM. 


\begin{tabular}{|c|c|c|c|c|c|c|c|c|}
\hline \multirow{3}{*}{ Network } & $\begin{array}{c}\text { \# } \\
\text { traffic } \\
\text { requests }\end{array}$ & $\begin{array}{c}\text { \# slots } \\
\text { primary } \\
\text { lightpaths }\end{array}$ & \multicolumn{2}{|c|}{$\begin{array}{c}\text { \# generated } \\
\text { columns } \\
\text { CG_DP }\end{array}$} & \multicolumn{2}{|c|}{$z_{\text {LP }}$} & \multicolumn{2}{|c|}{$\tilde{z}_{\text {ILP }}$} \\
& 20 & 164 & 8,735 & 12,875 & CG_DP & CG_SP & CG_DP & CG_SP \\
\hline \multirow{3}{*}{ nobel-US } & 40 & 273 & 15,190 & 21,744 & 546 & 171.05 & 292 & 201 \\
& 60 & 457 & 19,128 & 28,316 & 816 & 328.82 & 546 & 290 \\
& 40 & 344 & 26,828 & 40,931 & 574 & 339.6 & 574 & 430 \\
\hline \multirow{3}{*}{ USnet } & 80 & 856 & 39,514 & 67,936 & 1,278 & 557.37 & 1,278 & 713 \\
& 120 & 1138 & 46,938 & 80,495 & 1,790 & 835.55 & 1,790 & 1,021 \\
\hline
\end{tabular}

TABLE I: Numerical results for CG_DP and CG_SP.

Performance of CG Models. Table I summarizes the results of the two decomposition models for dedicated and shared protection on the two considered networks. We considered different numbers of demands. The load of each demand is randomly selected according to a uniform distribution within $50-200 \mathrm{~Gb} / \mathrm{s}$.

A first difference can be observed in the number of generated columns, revealing the different level of complexity of the two models. This has an impact on the completion time, as can be observed in Figure 2. The large number of generated columns is also a consequence of our solving strategy. In fact, in order to accelerate the time needed to solve the RMP and to find an ILP solution to the last RMP, at each iteration, we remove nonbasic columns from the master problem according to their marginal cost. Thus, the number of iterations increases but, on the other hand, the time needed to find a solution decreases. Another difference between the two models is the quality of the solution. CG_DP may require twice the number of frequency slots than CG_SP. This is a natural consequence of the different protection strategies. Moreover, the two models exhibit a different level of accuracy as expressed by the ratio of $\left(\tilde{z}_{\mathrm{LP}}-z_{\mathrm{LP}}\right) / z_{\mathrm{LP}}$. In the case of CG_DP, it never exceeds $1 \%$, while, for CG_SP, it may go up to $20 \%$. The main reason for the difference in accuracy of the two models is the following. In CG_DP, to reduce the spectrum usage, the goal is to try to use short paths. This leads to fractional solutions with a small number of paths (and often a single one) for each demand. On the contrary, in CG_SP, the goal is to share backup paths as much as possible in order to reduce the value of the objective function. This leads to a large number of fractional paths per demand (sharing frequency slots with backup paths of several other demands) in the optimal fractional solution. The last RMP thus contains a large number of path variables with a nonzero value (often $<0.1$ ) for each demand. Only one of them will be set to 1 per demand, when solving the last RMP as an ILP, leading to a larger gap.

Shared vs. Dedicated Path Protection. We now compare the performances of the two protection schemes. In Figure 3, we study the impact of the number of demands on the resources required by the two protection schemes. We keep the total traffic intensity constant and vary the number of demands. The traffic is set to be 10 Tbps on nobel-US and 15 Tbps on USnet. As the results indicate, the two protection schemes exhibit a very different behavior. As the number of demands increases, the performance of the shared protection scheme, defined in terms of used frequency slots improves. On the other hand, both the primary lightpaths and the backup lightpaths computed according to the dedicated protection scheme, tend to require more resources as the number of demands becomes larger. This is not surprising, since an increasing number of demands improves the frequency slots' sharing opportunities of the lightpaths. In fact, in the shared protection scheme two link-disjoint primary lightpaths may share frequency slots

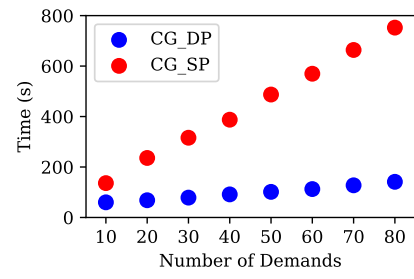

(a) nobel-US

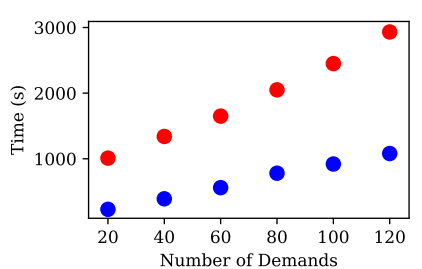

(b) USnet
Fig. 2: Average completion time as a function of the number of demands

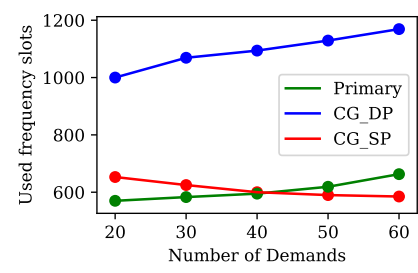

(a) nobel-US

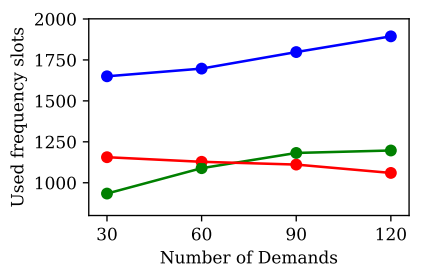

(b) USnet
Fig. 3: Average number of frequency slots used as a function of the number of demands

in their backup paths. The benefits of shared over dedicated path protection is about $20 \%$ and $40 \%$ in the two networks according to the number of demands. Indeed, the benefits tend to increase with the number of considered demands. These results are similar to the ones reported by [12] and [13].

Regenerators and Modulation Formats. Since, in optical networks, regenerators are costly, we are interested in evaluating the impact of the number of regenerators on the lightpaths. In Figures 4 and 5, we study the impact of the number of regenerators on the paths' latencies and on the spectrum requirements for the protection. We consider 50 demands for nobel-US and 100 demands on USnet. As the number of nodes with regeneration capabilities increases, from 0 to 10 for nobel-US and from 5 to 15 for USnet (Fig. 5), the spectrum requirements of the primary lightpaths and of the backup lightpaths decrease in both protection schemes. The reason is that a higher number of regenerators allows the lightpaths to use better modulation formats (in terms of bits per symbol) and consequently to use fewer resources. However, when considering lightpaths' latencies, the two protection schemes behave surprisingly in a strikingly different way. While, in the dedicated protection case, backup lightpaths' latencies tend to decrease, in the shared protection case, we observe the reverse phenomena. The explanation is the following. In dedicated protection, backup paths cannot be shared and, thus, the only means to reduce the number of used frequency slots is to use shorter paths. This is what happens when increasing the number of regenerators. Indeed, both primary and backup lightpaths need fewer resources, as they may now use more efficient modulation formats. This leads to increased spare capacity, allowing backup paths to use shorter routes. In shared protection, the situation is different. Indeed, there are two ways to reduce the spectrum usage: shorter paths as for DP, but also increased sharing of backup paths. The second way happens to be predominant in our experiments: regenerators allow better 


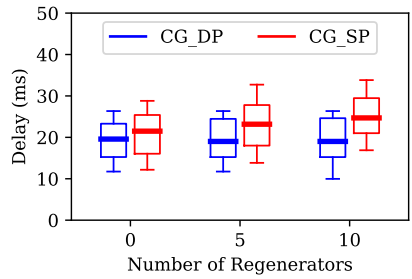

(a) nobel-US

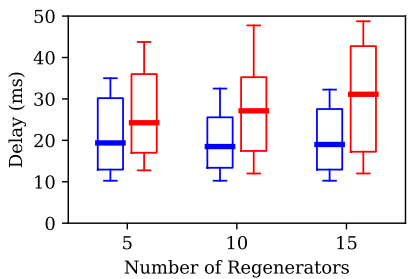

(b) USnet
Fig. 4: Path delay distributions under the two protection schemes vs. the number of regenerators.

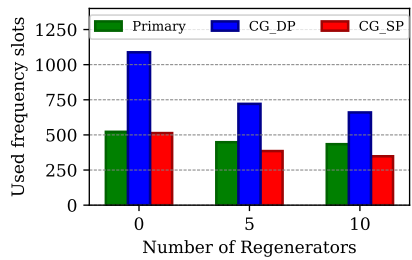

(a) nobel-US

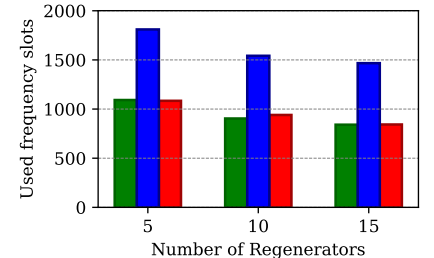

(b) USnet
Fig. 5: Average number of frequency slots used as a function of the number of regenerators

modulation formats and longer routes, leading to better sharing opportunities. As a consequence, the spectrum requirements are reduced, but this comes at the cost of increased lightpath lengths. However, the maximum delay of the backup paths in the shared protection case never exceeds $50 \mathrm{~ms}$, the value often chosen as the maximum allowed delay for a route in networks [24]. As the results indicate, particular attention should be paid to lightpaths' latencies when considering shared path protection, in order not to violate the SLA requirements. Indeed, with the spectrum resources as optimization task, the possibility to share resources may lead to longer paths at the expense of the delay. Note that we could also easily add a constraint in the pricing problem in order to consider only lightpaths under a certain delay requirement.

\section{CONCLUSION}

In this paper, we investigated the problem of providing path protection against a single link failure in elastic optical networks. We presented two decomposition models for both dedicated and shared path protection schemes taking into consideration modulation, regenerators, and shared risk link group constraints. Through extensive simulation, we showed the effectiveness of our models in finding a solution in a reasonable amount of time. Moreover, we studied different metrics in order to compare the accuracy of those models, showing the tradeoff in terms of required bandwidth and latency with the time resources needed by the two protection schemes. Our future works include the further improvement of the model precision and scalability, in order to be able to deal with larger and more complex instances of the problem.

\section{REFERENCES}

[1] M. Jinno, H. Takara, B. Kozicki, Y. Tsukishima, Y. Sone, and S. Matsuoka, "Spectrum-efficient and scalable elastic optical path network: ar- chitecture, benefits, and enabling technologies," IEEE Communications Magazine, vol. 47, no. 11, 2009.

[2] M. Jinno, B. Kozicki, H. Takara, A. Watanabe, Y. Sone, T. Tanaka, and A. Hirano, "Distance-adaptive spectrum resource allocation in spectrum-sliced elastic optical path network [topics in optical communications]," IEEE Communications Magazine, vol. 48, no. 8, 2010.

[3] M. Klinkowski and K. Walkowiak, "Routing and spectrum assignment in spectrum sliced elastic optical path network," IEEE Communications Letters, vol. 15, no. 8, pp. 884-886, 2011.

[4] D. Turner, K. Levchenko, A. C. Snoeren, and S. Savage, "California fault lines: understanding the causes and impact of network failures," in ACM SIGCOMM Computer Communication Review, 2010.

[5] G. Iannaccone, C.-n. Chuah, R. Mortier, S. Bhattacharyya, and C. Diot, "Analysis of link failures in an ip backbone," in Proceedings of the 2nd ACM SIGCOMM Workshop on Internet measurment. ACM, 2002.

[6] S. Ramamurthy, L. Sahasrabuddhe, and B. Mukherjee, "Survivable wdm mesh networks," Journal of Lightwave Technology, vol. 21, no. 4, 2003.

[7] L. Sahasrabuddhe, S. Ramamurthy, and B. Mukherjee, "Fault management in ip-over-wdm networks: Wdm protection versus ip restoration," IEEE journal on selected areas in communications, vol. 20, no. 1, 2002.

[8] D. Zhou and S. Subramaniam, "Survivability in optical networks," IEEE network, vol. 14, no. 6, pp. 16-23, 2000.

[9] M. Klinkowski and K. Walkowiak, "Offline rsa algorithms for elastic optical networks with dedicated path protection consideration," in Ultra Modern Telecommunications and Control Systems and Workshops (ICUMT), 2012 4th International Congress on. IEEE, 2012.

[10] M. Klinkowski, "An evolutionary algorithm approach for dedicated path protection problem in elastic optical networks," Cybernetics and Systems, vol. 44, no. 6-7, pp. 589-605, 2013.

[11] _ "A genetic algorithm for solving rsa problem in elastic optical networks with dedicated path protection," in International Joint Conference CISIS12-ICEUTE' 12-SOCO' 12 Special Sessions. Springer, 2013, pp. 167-176.

[12] G. Shen, Y. Wei, and S. K. Bose, "Optimal design for shared backup path protected elastic optical networks under single-link failure," Journal of Optical Communications and Networking, vol. 6, no. 7, 2014.

[13] K. Walkowiak and M. Klinkowski, "Shared backup path protection in elastic optical networks: Modeling and optimization," in Design of Reliable Communication Networks (DRCN), 2013 9th International Conference on the. IEEE, 2013, pp. 187-194.

[14] M. Ruiz, M. Pióro, M. Żotkiewicz, M. Klinkowski, and L. Velasco, "Column generation algorithm for rsa problems in flexgrid optical networks," Photonic network communications, vol. 26, no. 2-3, 2013.

[15] K. Christodoulopoulos, I. Tomkos, and E. Varvarigos, "Elastic bandwidth allocation in flexible ofdm-based optical networks," Journal of Lightwave Technology, vol. 29, no. 9, pp. 1354-1366, 2011.

[16] A. Bocoi, M. Schuster, F. Rambach, M. Kiese, C.-A. Bunge, and B. Spinnler, "Reach-dependent capacity in optical networks enabled by ofdm," in Proc. Optical Fiber Communication (OFC), 2009. IEEE.

[17] A. Kretsis, K. Christodoulopoulos, P. Kokkinos, and E. Varvarigos, "Planning and operating flexible optical networks: Algorithmic issues and tools," IEEE Communications Magazine, vol. 52, no. 1, 2014.

[18] V. Chvatal, Linear Programming. Freeman, 1983.

[19] M. R. Garey and D. S. Johnson, Computers and intractability. wh freeman New York, 2002, vol. 29.

[20] S. Irnich and G. Desaulniers, "Shortest path problems with resource constraints," Column generation, pp. 33-65, 2005.

[21] S. Orlowski, R. Wessäly, M. Pióro, and A. Tomaszewski, "Sndlib 1.0survivable network design library," Networks, vol. 55, no. 3, 2010.

[22] B. Mukherjee, Optical WDM networks. Springer Science \& Business Media, 2006.

[23] Z. Zhu, W. Lu, L. Zhang, and N. Ansari, "Dynamic service provisioning in elastic optical networks with hybrid single-/multi-path routing," Journal of Lightwave Technology, vol. 31, no. 1, pp. 15-22, 2013.

[24] F. Giroire, A. Nucci, N. Taft, and C. Diot, "Increasing the robustness of ip backbones in the absence of optical level protection," in INFOCOM 2003. IEEE, 2003, pp. 1-11. 